\title{
MacNeish-Mann Theorem Based Iteratively Decodable Codes for Optical Communication Systems
}

\author{
Ivan B. Djordjevic and Bane Vasic, Senior Member, IEEE
}

\begin{abstract}
A novel family of low-density parity-check codes is proposed based on MacNeish-Mann theorem for construction of mutually orthogonal Latin squares. Codes from this family have high code rate, girth at least six, large minimum distance, and significantly outperform conventional forward error correction schemes based on Reed-Solomon (RS) and turbo codes.
\end{abstract}

Index Terms-Forward error correction (FEC), low-density parity-check (LDPC) codes, MacNeish-Mann theorem, mutually orthogonal Latin squares (MOLS), optical communications.

\section{INTRODUCTION}

A DVANCED forward error correction (FEC) codes have started playing a key role in boosting performance of dense wavelength-division multiplexing (DWDM) systems in long-haul transmission. The coding gain allows to effectively increase the amplifier spacing, the transmission distance and/or data rates. The state-of-the-art optical communication systems standardized by the ITU all employ Bose-Chaudhuri-Hocquenghen $(\mathrm{BCH})$ codes [1]: ([4359, 4320] shortened BCH-3 code in the ITU-T G.707 standard, $[255,238]$ Reed-Solomon (RS) code in the digital wrapper of the ITU G.709 standard, and [255, 239] RS code in the ITU-T G.975 standard [2]). For optical communication systems with bit rates beyond $10 \mathrm{~Gb} / \mathrm{s}$ more powerful FEC codes with larger coding gains and high code rates are necessary. Two examples of such code include a concatenated scheme with two RS codes proposed by Ait Sab et al. [3], and block turbo codes proposed by Pyndiah [4] and Ait Sab et al. [5].

Recently, we showed in [6]-[8] that the error performance and hardware complexity of turbo codes can be outperformed by low-density parity check (LDPC) codes. LDPC codes can be designed in a pseudorandom fashion [9], but this leads to encoders/decoders complexity that exceeds practical limits imposed on high-speed optical communication systems. A more attractive approach applied here is to design LDPC codes based on combinatorial objects that allow encoding and decoding to

\footnotetext{
Manuscript received December 16, 2003. The associate editor coordinating the review of this letter and approving it for publication was Prof. C.-C. Chao. This work is supported by the National Science Foundation under Grant ITR 0325979 and Grant CCR 0208597.

I. B. Djordjevic is with the Department of Electrical and Computer Engineering, University of Arizona, Tucson, AZ 85721 USA, on leave from the Faculty of Computing, Engineering and Mathematical Sciences, University of the West of England, Bristol, U.K. (e-mail: ivan@ece.arizona.edu).

B. Vasic is with the Department of Electrical and Computer Engineering, University of Arizona,Tucson, AZ 85721 USA (e-mail: vasic@ece.arizona.edu).

Digital Object Identifier 10.1109/LCOMM.2004.833833
}

be performed via fast and simple circuits (no permuter is necessary and the parity-check matrix has quasi-cyclic structure, see [8], for more details). Such high-speed FEC architectures are of crucial importance in optical communications, and there has been a great deal of activity in this area. For example, Agere Systems (see Azadet et al. [10]) reported an optical networking interface device with four parallel RS codecs, each operating at $2.5 \mathrm{~Gb} / \mathrm{s}$. Agere Systems (see [11], [12]) has also implemented a rate $1 / 2$ block length 1024 LDPC code for optical networking, and Flarion [13] researchers developed codecs employing proprietary multi-edge type LDPC code of length 4096.

In this letter, a novel class of LDPC codes based on mutually orthogonal Latin squares (MOLS) constructed using MacNeish-Mann theorem is proposed. These codes have high code rate, large minimum distance, girth of at least six, low complexity itereative decoding algorithm and excellent BER performance. The performance of the proposed codes is assessed in a very realistic simulation environment (for more details the reader is referred to [7]) that includes impairments originating from amplified spontaneous emission (ASE) noise, pulse distortions due to fiber nonlinearities, chromatic dispersion, crosstalk effects, and intersymbol interference. We show that the coding gain of these LDPC codes is significantly higher than the coding gain of RS codes of larger redundancy and higher than coding gain of turbo codes of larger redundancy, which makes them excellent candidates for use in long-haul transmission systems.

\section{CODE DESCRIPTION}

The codes proposed in this paper are based on the theory of MOLS constructed using MacNeish-Mann theorem [15].

Recently [8], we gave a construction of LDPC codes using MOLS. In this section, we explain the method of constructing the complete system of MOLS (adopted from [15]) for any composite integer $q$, which generalizes our previous construction [8].

Let the prime decomposition of an integer $q$ be

$$
q=p_{1}^{s_{1}} p_{2}^{s_{2}} \cdots p_{m}^{s_{m}}
$$

with $p_{i}(i=1,2, \ldots, m)$ being the prime numbers. MacNeish and Mann showed [15] that a complete set of

$$
n(q)=\min \left(p_{1}^{s_{1}}, p_{2}^{s_{2}}, \ldots, p_{m}^{s_{m}}\right)-1 .
$$

MOLS of order $q$ can always be constructed.

The construction algorithm follows. Consider $m$-tuples

$$
\gamma=\left(g_{1}, g_{2}, \ldots, g_{m}\right)
$$


where $g_{i} \in G F\left(p_{i}^{s_{i}}\right), i=1,2, \ldots, m$, with addition and multiplication operations defined by

$$
\begin{aligned}
\gamma_{1}+\gamma_{2} & =\left(g_{1}, g_{2}, \ldots, g_{m}\right)+\left(h_{1}, h_{2}, \ldots, h_{m}\right) \\
& =\left(g_{1}+h_{1}, g_{2}+h_{2}, \ldots, g_{m}+h_{m}\right) \\
\gamma_{1} \gamma_{2} & =\left(g_{1} h_{1}, g_{2} h_{2}, \ldots, g_{m} h_{m}\right)
\end{aligned}
$$

wherein the operations in brackets are performed in the corresponding Galois fields (e.g., $g_{1} h_{1}$ and $g_{1}+h_{1}$ are performed in $\left.G F\left(p_{1}^{s_{1}}\right)\right)$. Denote the elements from $G F\left(p_{i}^{s_{i}}\right)$, $i=1,2, \ldots, m$ as $0, g_{i}^{(1)}=1, g_{i}^{(2)}, \ldots, g_{i}^{\left(p_{i}^{s_{i}}-1\right)}$. The following elements possesses multiplicative inverses:

$$
\gamma_{i}=\left(g_{1}^{(i)}, g_{2}^{(i)}, \ldots, g_{m}^{(i)}\right), \quad i=1,2, \ldots, n(q)
$$

since they are composed of nonzero elements of corresponding Galois fields, and $\gamma_{i}-\gamma_{j}$ for $i \neq j$ as well. Denote the first element as $\gamma_{0}=(0,0, \ldots, 0)$, the next $n(q)$ as in (6), and the rest $q-n(q)-1$ elements as

$$
\begin{aligned}
\gamma_{j} & =\left(g_{1}^{\left(i_{1}\right)}, g_{2}^{\left(i_{2}\right)}, \ldots, g_{m}^{\left(i_{m}\right)}\right), \quad j=n(q)+1, \ldots, q-1 \\
i_{l} & =0,1, \ldots, p_{l}^{s_{l}}-1, \quad l=1,2, \ldots, m
\end{aligned}
$$

with combinations of components leading to (6) being excluded.

The $n(q)$ arrays of elements, MOLS, $L_{i}, i=1,2, \ldots, n(q)$ are further formed with $(j, k)$ th cell being filled by $\gamma_{j}+\gamma_{i} \gamma_{k}$; $i=1,2, \ldots, n(q) ; 0 \leq j, k \leq q-1$. (The multiplication $\gamma_{i} \gamma_{k}$ is performed using (5), and the addition $\gamma_{j}+\gamma_{i} \gamma_{k}$ using (4).) To establish the connection between MOLS and BIBD, we introduce the following one-to-one mapping $l: L \rightarrow V$, with $L$ being the MOLS and $V$ being the integer set of $q^{2}$ elements (points) (for more details on BIBDs please refer to [8], [14]). The simplest example of such mapping is the linear mapping $l(x, y)=q(x-1)+y ; 1 \leq x, y \leq q$ (therefore, $1 \leq l(x, y) \leq$ $q^{2}$ ). (The numbers $l(x, y)$ are referred to as the cell labels.) Each $L_{i}(i=1, \ldots, n(q))$ defines a set of $q$ blocks (lines) $B_{i}=$ $\left\{l(x, y) \mid L_{i}(x, y)=s, 1 \leq s \leq q\right\}$. Each line $s(1 \leq s \leq q)$ in $B_{i}$ contains the labels of element $s$ from $L_{i}$. These blocks are equivalent to the sets of lines of slopes $0 \leq s \leq q-1$ in the lattice design introduced in our earlier paper [6]. Labels of every line of a design specify the positions of " 1 "s in a row of parity check matrix. Namely, the incidence matrix of a design is a $q n(q) \times q^{2}$ matrix $H=\left(h_{i j}\right)$ defined by

$$
h_{i j}= \begin{cases}1, & \text { if the } i \text { th block contains the } j \text { th point } \\ 0, & \text { otherwise }\end{cases}
$$

and represents the parity-check matrix. The code length is $q^{2}$, the code rate is determined by $R=\left(q^{2}-\operatorname{rank}(H)\right) / q^{2}$, and may be estimated as $R \cong\left(q^{2}-n(q) q\right) / q^{2}=1-n(q) / q$.

Notice that when $q$ is a prime power, the construction proposed here reduces to our previous construction [8].

Finding the minimum distance of BIBD codes is difficult in general. However, it can be shown that a lower bound on minimum distance is $q+1$.

Example: For $q=12=2^{2} \cdot 3^{1}$ the number of MOLS is $n(q)=2$, and corresponding $\gamma$-elements are:

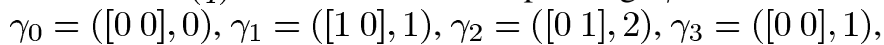

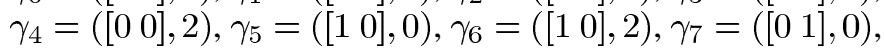
$\gamma_{8}=\left(\left[\begin{array}{ll}0 & 1\end{array}\right], 1\right), \gamma_{9}=\left(\left[\begin{array}{ll}1 & 1\end{array}\right], 0\right), \gamma_{10}=\left(\left[\begin{array}{ll}1 & 1\end{array}\right], 1\right)$, and $\gamma_{11}=([11], 2)$. (Primitive polynomials of $\mathrm{GF}\left(2^{2}\right)$ and $\mathrm{GF}(3)$ used in this example are $1+x+x^{2}$ and $1+x$, respectively.) The two MOLS are (the elements of MOLS instead of $\gamma_{0}, \gamma_{1}, \ldots, \gamma_{11}$ are denoted by integers $1,2, \ldots, q=12$ ); respectively

$$
\begin{aligned}
L_{1} & =\left[\begin{array}{cccccccccccc}
1 & 2 & 3 & 4 & 5 & 6 & 7 & 8 & 9 & 10 & 11 & 12 \\
2 & 5 & 10 & 7 & 6 & 4 & 1 & 11 & 12 & 9 & 3 & 8 \\
3 & 10 & 4 & 8 & 9 & 12 & 11 & 5 & 1 & 7 & 6 & 2 \\
4 & 7 & 8 & 5 & 1 & 2 & 6 & 9 & 3 & 11 & 12 & 10 \\
5 & 6 & 9 & 1 & 4 & 7 & 2 & 3 & 8 & 12 & 10 & 11 \\
6 & 4 & 12 & 2 & 7 & 1 & 5 & 10 & 11 & 8 & 9 & 3 \\
7 & 1 & 11 & 6 & 2 & 5 & 4 & 12 & 10 & 3 & 8 & 9 \\
8 & 11 & 5 & 9 & 3 & 10 & 12 & 1 & 4 & 6 & 2 & 7 \\
9 & 12 & 1 & 3 & 8 & 11 & 10 & 4 & 5 & 2 & 7 & 6 \\
10 & 9 & 7 & 11 & 12 & 8 & 3 & 6 & 2 & 1 & 4 & 5 \\
11 & 3 & 6 & 12 & 10 & 9 & 8 & 2 & 7 & 4 & 5 & 1 \\
12 & 8 & 2 & 10 & 11 & 3 & 9 & 7 & 6 & 5 & 1 & 4
\end{array}\right] \\
L_{2} & =\left[\begin{array}{ccccccccccccc}
1 & 3 & 11 & 5 & 4 & 8 & 9 & 10 & 12 & 6 & 7 & 2 \\
2 & 10 & 3 & 6 & 7 & 11 & 12 & 9 & 8 & 4 & 1 & 5 \\
3 & 4 & 6 & 9 & 8 & 5 & 1 & 7 & 2 & 12 & 11 & 10 \\
4 & 8 & 12 & 1 & 5 & 9 & 3 & 11 & 10 & 2 & 6 & 7 \\
5 & 9 & 10 & 4 & 1 & 3 & 8 & 12 & 11 & 7 & 2 & 6 \\
6 & 12 & 9 & 7 & 2 & 10 & 11 & 8 & 3 & 1 & 5 & 4 \\
7 & 11 & 8 & 2 & 6 & 12 & 10 & 3 & 9 & 5 & 4 & 1 \\
8 & 5 & 2 & 3 & 9 & 1 & 4 & 6 & 7 & 10 & 12 & 11 \\
9 & 1 & 7 & 8 & 3 & 4 & 5 & 2 & 6 & 11 & 10 & 12 \\
10 & 7 & 4 & 12 & 11 & 6 & 2 & 1 & 5 & 8 & 3 & 9 \\
11 & 6 & 5 & 10 & 12 & 2 & 7 & 4 & 1 & 9 & 8 & 3 \\
12 & 2 & 1 & 11 & 10 & 7 & 6 & 5 & 4 & 3 & 9 & 8
\end{array}\right] .
\end{aligned}
$$

Blocks of the corresponding BIBD are given as rows of the array $B$

$$
B=\left[\begin{array}{cccccccccccc}
1 & 19 & 33 & 41 & 52 & 66 & 74 & 92 & 99 & 118 & 132 & 143 \\
2 & 13 & 36 & 42 & 55 & 64 & 77 & 95 & 106 & 117 & 128 & 135 \\
3 & 23 & 25 & 45 & 56 & 72 & 82 & 89 & 100 & 115 & 122 & 138 \\
4 & 18 & 27 & 37 & 53 & 62 & 79 & 93 & 104 & 119 & 130 & 144 \\
5 & 14 & 32 & 40 & 49 & 67 & 78 & 87 & 105 & 120 & 131 & 142 \\
6 & 17 & 35 & 43 & 50 & 61 & 76 & 94 & 108 & 116 & 123 & 141 \\
7 & 16 & 34 & 38 & 54 & 65 & 73 & 96 & 107 & 111 & 129 & 140 \\
8 & 24 & 28 & 39 & 57 & 70 & 83 & 85 & 101 & 114 & 127 & 134 \\
9 & 22 & 29 & 44 & 51 & 71 & 84 & 88 & 97 & 110 & 126 & 139 \\
10 & 15 & 26 & 48 & 59 & 68 & 81 & 90 & 103 & 109 & 125 & 136 \\
11 & 20 & 31 & 46 & 60 & 69 & 75 & 86 & 102 & 112 & 121 & 137 \\
12 & 21 & 30 & 47 & 58 & 63 & 80 & 91 & 98 & 113 & 124 & 133 \\
1 & 23 & 31 & 40 & 53 & 70 & 84 & 90 & 98 & 116 & 129 & 135 \\
12 & 13 & 33 & 46 & 59 & 65 & 76 & 87 & 104 & 115 & 126 & 134 \\
2 & 15 & 25 & 43 & 54 & 69 & 80 & 88 & 101 & 119 & 132 & 142 \\
5 & 22 & 26 & 37 & 52 & 72 & 83 & 91 & 102 & 111 & 128 & 141 \\
4 & 24 & 30 & 41 & 49 & 71 & 82 & 86 & 103 & 117 & 123 & 140 \\
10 & 16 & 27 & 47 & 60 & 61 & 77 & 92 & 105 & 114 & 122 & 139 \\
11 & 17 & 32 & 48 & 58 & 64 & 73 & 93 & 99 & 110 & 127 & 138 \\
6 & 21 & 29 & 38 & 55 & 68 & 75 & 85 & 100 & 118 & 131 & 144 \\
7 & 20 & 28 & 42 & 50 & 63 & 81 & 89 & 97 & 120 & 130 & 143 \\
8 & 14 & 36 & 45 & 51 & 66 & 79 & 94 & 107 & 109 & 124 & 137 \\
3 & 18 & 35 & 44 & 57 & 67 & 74 & 96 & 106 & 113 & 121 & 136 \\
9 & 19 & 34 & 39 & 56 & 62 & 78 & 95 & 108 & 112 & 125 & 133
\end{array}\right]
$$

For example, the first row in $B$ denotes the labels of element 1 from $L_{1}$. (The labels in the first row of $L_{1}$ run from 1 to 12 , in the second from 13 to 24 , etc.) The rows of $B$ specify positions of nonzero elements in the rows of a parity-check matrix (elements of $B$ run from 1 to $q^{2}$ ). 


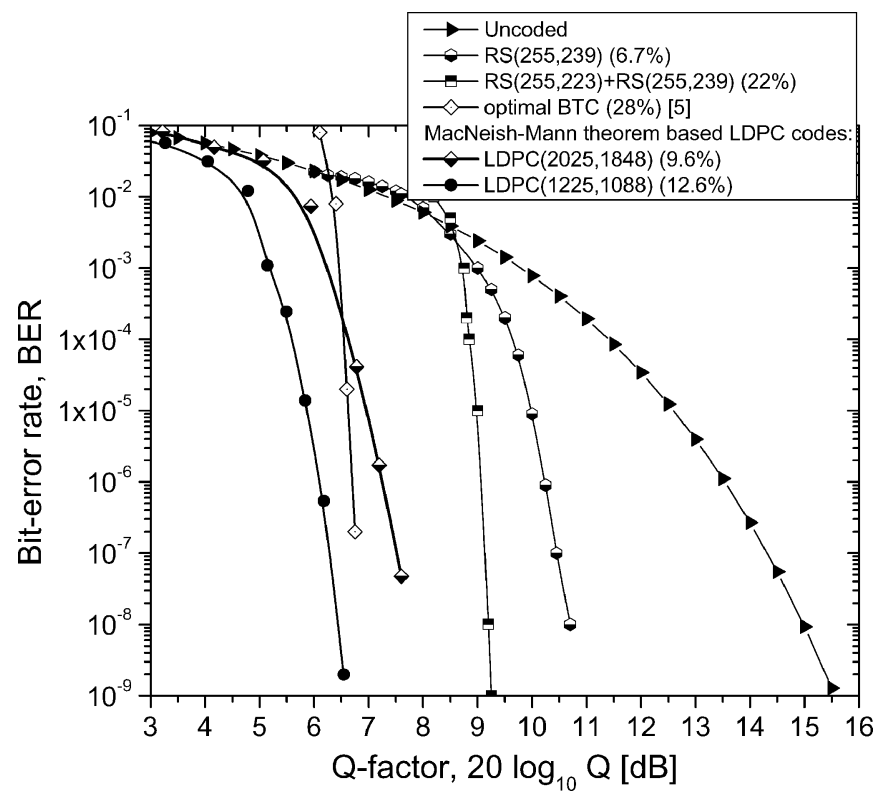

Fig. 1. BER performance of LDPC codes on MacNeish-Mann MOLS at 40 $\mathrm{Gb} / \mathrm{s}$ with six iterations in min-sum algorithm.

\section{PERformance ANALYSIS}

Fig. 1 plots the BER results obtained from Monte Carlo simulations with six iterations in the min-sum decoding algorithm for a MOLS (using MacMeish-Mann theorem for $q=45)$ LDPC $[2025,1848]$ code with code rate $R=0.913$ (redundancy of $9.57 \%$ ), and a MOLS based LDPC [1225, 1088] with code rate 0.888 (redundancy of $12.59 \%$ ), constructed for $q=35$. (Notice that here we use the definition of redundancy commonly used for optical communications; see e.g., [16].) A WDM system with $40-\mathrm{Gb} / \mathrm{s}$ bit-rate per channel and a channel spacing of $100 \mathrm{GHz}$ is considered. It is assumed that the observed channel is located at $1552.524 \mathrm{~nm}(193.1 \mathrm{THz})$ and that there exists a nonnegligible interaction with six neighboring channels. The influence of optical and electrical filters is taken into account as well. The dispersion map of considered transmission system is composed of a SMF section (of length $80 \mathrm{~km}$ ), followed by an EDFA to compensate the fiber losses, and a DCF section to compensate both GVD and second-order GVD, as well as another EDFA to compensate the fiber losses in corresponding DCF section. Eight SMF-DCF sections are observed, and $Q$-factor is additionally decreased by noise loading. The average power per channel is set to be $0 \mathrm{dBm}$, and the carrier-suppressed RZ signal format is assumed. (The channel characteristics modeled according to our previous papers [7] are used to create samples fed to iterative decoder.) Using the expression

$$
\text { Uncoded_BER }=\left(\frac{1}{2}\right) \text { erfc }\left(\frac{Q}{\sqrt{2}}\right)
$$

the bit-error rate of uncoded signal is converted to $Q$-factor to make the results comparable with previously reported ones.
The LDPC code of redundancy $9.57 \%$ significantly outperforms $\operatorname{RS}[255,223]+\operatorname{RS}[255,239]$ concatenation scheme [5], while LDPC code of redundancy $12.59 \%$ outperforms the optimized block turbo code [5] of redundancy $28 \%$. Notice the complexity of the turbo decoding algorithm is higher than that of a decoder based on min-sum algorithm. (Encoding complexity and the decoding hardware complexity of BIBD codes are discussed in [17].)

\section{CONCLUSION}

We proposed a novel class of LDPC codes, with high code rate, large minimum distance and girth at least six, based on MOLS constructed using MacNeish-Mann theorem.

The proposed LPDC codes perform very well in the presence of ASE noise, fiber nonlinearities, chromatic dispersion, and intersymbol interference. Since the proposed FEC scheme significantly outperforms traditional FEC schemes based on $\mathrm{RS}$ and turbo codes it is an excellent candidate for long-haul transmission.

\section{REFERENCES}

[1] S. Lin and D. J. Costello, Error Control Coding: Fundamentals and Applications. Englewood Cliffs, NJ: Prentice-Hall, 1983.

[2] "ITU, Telecommunication Standardization Sector Forward Error Correction for Submarine Systems,", Tech. Recommendation G.975/ G709.

[3] O. A. Sab et al., "Concatenated forward error correction schemes for long-haul DWDM optical transmission systems," in ECOC, 1999, pp. II-290-II-290.

[4] R. M. Pyndiah, "Near optimum decoding of product codes," IEEE Trans. Commun., vol. 46, pp. 1003-1010, Aug. 1998.

[5] O. A. Sab and V. Lemarie, "Block turbo code performances for long-haul DWDM optical transmission systems," in Proc. Optical Fiber Communication Conf. (OFC), vol. 3, 2001, pp. 280-282.

[6] B. Vasic and I. B. Djordjevic, "Low-Density parity check codes for long haul optical communications systems," IEEE Photon. Technol. Lett., vol. 14, pp. 1208-1210, 2002.

[7] I. B. Djordjevic and B. Vasic, "Projective geometry low-density paritycheck codes for ultra-long haul WDM high-speed transmission," IEEE Photon. Technol. Lett., vol. 15, pp. 784-786, May 2003.

[8] B. Vasic, I. B. Djordjevic, and R. Kostuk, "Low-density parity check codes and iterative decoding for long haul optical communication systems," J. Lightwave Technol., vol. 21, pp. 438-446, Feb. 2003.

[9] D. J. C. MacKay, "Good error correcting codes based on very sparse matrices," IEEE Trans. Inform. Theory, pp. 399-431, Mar. 1999.

[10] K. Azadet et al., "Equalization and FEC techniques for optical transceivers," IEEE J. Solid-State Circuits, vol. 37, pp. 317-327, Mar. 2002.

[11] C. Howland and A. Blanksby, "A $220 \mathrm{~mW} 1 \mathrm{~Gb} / \mathrm{s}$ 1024-Bit rate-1/2 low density parity check code decoder," in IEEE Conf. Custom Integ. Circuits, May 2001, pp. 293-296.

[12] — , "Parallel decoding architectures for low density parity check codes," in ISCAS 2001, vol. 4, May 2001, pp. 742-745.

[13] Flarion. Vector LDPC technology. [Online]. Available: http://www. flarion.com/products/products_vector.html

[14] I. Anderson, Combinatorial Designs: Construction Methods. Chichester, U.K.: Ellis Horwood Ltd., 1990.

[15] D. Raghavarao, Constructions and Combinatorial Problems in Design of Experiments. New York: Dover, 1988.

[16] O. A. Sab, "Forward error correction for optical transmission systems," in Proc. Optical Fiber Communication Conf. (OFC 2003), Atlanta, GA, 2003, pp. 83-104.

[17] R. Lynch et al., "The search for a practical iterative detector for magnetic recording," in TMRC 2003.

[18] M. Akita et al., "Third generation FEC employing turbo product code for long-haul DWDM transmission systems," in Tech. Dig. Optical Fiber Communication Conf. (OFC 2002), Anaheim, CA, 2002, paper WP2. 